\title{
Palmprint Recognition Based on Convolutional Neural Network-Alexnet
}

\author{
Weiyong Gong \\ School of Electronics and \\ Information Engineering MOE Key \\ Lab for Intelligent Networks and \\ Network Security Xi' an Jiaotong \\ University No.28 xianning west \\ road Xi'an, China \\ gong_w_y@stu.xjtu.edu.cn \\ Xuebin Xu \\ Guangdong Xi'an Jiaotong \\ University Academy. No. 3, \\ Daliangshuxiang East Road \\ Foshan, China \\ ccp9999@126.com.
}

\author{
Xinman Zhang \\ School of Electronics and \\ Information Engineering MOE Key \\ Lab for Intelligent Networks and \\ Network Security Xi'an Jiaotong \\ University No.28 xianning west \\ road Xi'an, China \\ zhangxinman@mail.xjtu.edu.cn
}

\author{
Bohua Deng \\ School of Electronics and \\ Information Engineering MOE Key \\ Lab for Intelligent Networks and \\ Network Security Xi'an Jiaotong \\ University No.28 xianning west \\ road Xi'an, China \\ bohuadeng@qq.com
}

\begin{abstract}
In the classic algorithm, palmprint recognition requires extraction of palmprint features before classification and recognition, which will affect the recognition rate. To solve this problem, this paper uses the convolutional neural network $(\mathrm{CNN})$ structure Alexnet to realize palmprint recognition. First, according to the characteristics of the geometric shape of palmprint, the ROI area of palmprint was cut out. Then the ROI area after processing is taken as input of convolutional neural network. Next the PRelu activation function is used to train the network to select the best learning rate and super parameters. Finally, the palmprint was classified and identified. The method was applied to PolyU Multi-Spectral Palmprint Image Database and PolyU 2D+3D Palmprint Database, and the recognition rate of a single spectrum was up to $99.99 \%$.
\end{abstract}

\section{INTRODUCTION}

W ITH the development of network and information technology, the society has put forward higher and higher requirements for the security of information systems. Biometric recognition technology has gradually become one of the important methods to enhance the security and stability of information systems. Biometrics is a technology that uses human physiology or behavioral features for automatic identification [1]. Biometrics are unique personal attributes, which have the characteristics of stability, diversity and individual differences. A number of biometrics have been used, including fingerprint, face, iris, signature,

This work was not supported by any organization finger vein, etc. [2]. Meanwhile, palmprint recognition technology is also developing rapidly [3].

Compared with other biological features, palmprint has many unique advantages, and each person's palmprint has different characteristics. Palmprint combines the texture and line features of the palm, and these features do not change over time. In comparison, the collected palmprint is easier to obtain rich personal information due to its larger area than fingerprint. Therefore, palmprint attracts more and more scholars' attention due to its advantages. Traditionally, researchers combine machine learning methods, such as SVM [4], KNN [5], etc., with feature extraction methods, such as LBP [6] and HOG [7], in palmprint recognition. Contrast to machine learning, deep learning achieves automatic feature extraction and classification.

Since 2012, the method based on deep convolutional neural network has achieved remarkable results in various computer vision tasks [9].In face recognition based on deep learning, researchers have made extensive research [10].In the aspect of palmprint recognition, researchers have carried out corresponding research experiments with the convolutional neural network method, and achieved good results [11].In this paper, Alexnet structure is adopted, and experiments and improvements are made on PolyU multispectral Database and PolyU 2D+3D Palmprint Database. The accuracy of each spectrum is quite good, which further proves the effectiveness of the deep learning method in Palmprint recognition.

The rest of the paper is organized as follows: Section II introduces the palmprint preprocessing method and the 
palmprint recognition method based on deep learning; Section III describes the experimental results obtained by verifying the algorithm on palmprint database; Section IV concludes the paper.

\section{METHODS}

\section{A. Image preprocessing}

Palmprint contains rich texture and structural features, such as the main line, wrinkles, triangulation and detail points. When collecting images, images of the same palmprint collected at different times will have different degrees of rotation and translation, and the size of palmprint collected at the same time may also be different. Therefore, before feature extraction and recognition of palmprint, it is necessary to extract the effective ROI area of palmprint containing main features. The whole processing process is shown in Fig.1. ROI extraction is a key step, and the correct ROI extraction is conducive to image alignment, improving the efficiency of feature matching, and finally giving a positive impact on the recognition results.

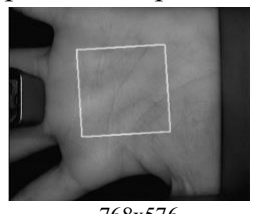

$768 \times 576$

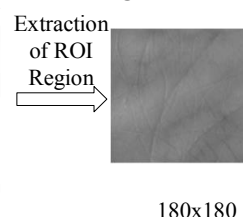

$180 \times 180$

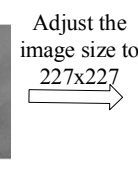
age size to $227 \times 227$

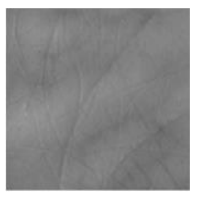

$227 \times 227$
Fig. 1 Image preprocessing

The relative position of the ROI area in the palm is fixed and located in the center of the palm. Zhang et al. proposed edge-based palmprint positioning processing method [12], which can accurately extract ROI images and thus has been widely used. This article uses this method to process the ROI area with rich features for the following part of the The ROI area of CASIA-Multi-Spectral- PalmprintV1 is shown in Fig. 2

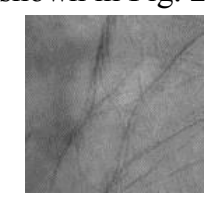

a)Blue

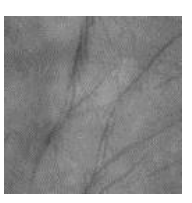

b)Green

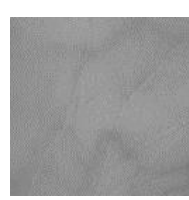

c)NIR

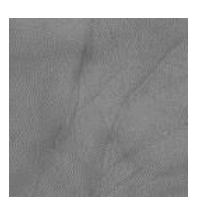

d)Red

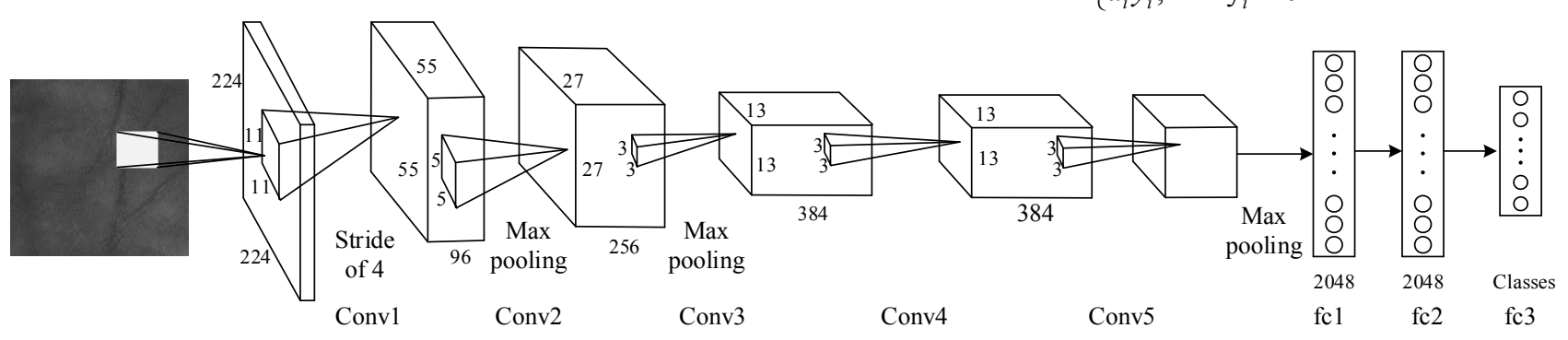

Fig. 3 Structure of Alexnet
Fig. 2 ROI region obtained by image preprocessing

\section{B. Convolutional Neural Network - Alexnet}

Convolutional neural network $(\mathrm{CNN})$ is a branch of deep learning which is widely studied and applied. It is not only a multi-layer network model, but also an improvement of BP neural network. They all use forward propagation to output calculated values, and use back propagation to adjust weights and biases. Different from the classical recognition algorithm, CNN repeatedly uses the convolution operation and pooling operation in the original input to obtain increasingly complex feature graphs, and finally directly outputs the results through the full connection. It mainly includes five parts which are input layer, convolution layer, pooling layer, full connection layer and output layer.

In this paper, Alexnet [9] is used and improved. It contains 8 layers of neural network, 5 convolution layers, 3 pooling layers and 3 full connection layers. The structure of the whole system is shown in table 1 and fig. 3 .

In Alexnet, the author used ReLU activation function and Dropout to improve speed and accuracy.Fig.4 (a) shows the ReLU activation function and the definition is shown in formula (1). Compared with Sigmoid and Tanh activation function, this activation function makes the network converge more rapidly. It can combat the gradient vanishing problem and has high calculation efficiency. When we use ReLU activation function, with the progress of training, there may be a situation where the neurons die and the weight cannot be updated. If that happens, then the gradient through the neuron from this point will always be zero.

$$
f(y)= \begin{cases}y, & \text { if } \mathrm{y}>0 \\ 0, & \text { if } \mathrm{y} \leq 0\end{cases}
$$

So, He [13] et al. proposed a new nonlinear correction activation function PReLU, whose definition is shown in formula (2).Compared with ReLU, PReLU converges faster. And although PReLU introduces extra parameter "a", it hardly needs to worry about overfitting. In addition, we can achieve learning updates of "a" through back propagation, which enables neurons to select the best gradient in the negative region. So in this article, we use PReLU as an activation function for Alexnet.

$$
f(y)= \begin{cases}y_{i}, & \text { if } \mathrm{y}_{i}>0 \\ a_{i} y_{i}, & \text { if } \mathrm{y}_{i} \leq 0\end{cases}
$$


TABLE I.

ARCHITECTURE OF ALEXNET

\begin{tabular}{|l|l|}
\hline \multicolumn{1}{|c|}{ Layers } & \multicolumn{1}{c|}{ Datails } \\
\hline Input & Image Input(227x227) \\
\hline Conv1 & 11x11 Convolutions with stride 4, padding 0 \\
\hline Relu 1 & PRelu \\
\hline Norm1 & Cross channel normalization \\
\hline Pool1 & 3x3 max pooling with stride 2 and padding 0 \\
\hline Conv2 & 5x5 Convolutions with stride 1, padding 2 \\
\hline Relu 2 & PRelu \\
\hline Norm2 & Cross channel normalization \\
\hline Pool2 & 3x3 max pooling with stride 2 and padding 0 \\
\hline Conv3 & 3x3 Convolutions with stride 1, padding 1 \\
\hline Relu 3 & PRelu \\
\hline Conv4 & 3x3 Convolutions with stride 1, padding 1 \\
\hline Relu 4 & PRelu \\
\hline Conv5 & 3x3 Convolutions with stride 1, padding 1 \\
\hline Relu 5 & PRelu \\
\hline Pool3 & 3x3 max pooling with stride 2 and padding 0 \\
\hline fc1 & 4096 fully connected layer \\
\hline Relu 6 & PRelu \\
\hline fc2 & 4096 fully connected layer \\
\hline Relu 6 & PRelu \\
\hline fc3 & n_classs fully connected layer \\
\hline softmax & softmax \\
\hline
\end{tabular}

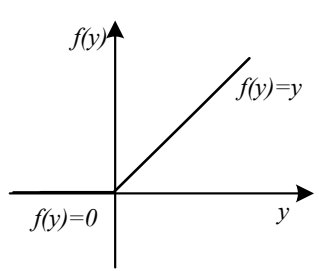

(a)Relu

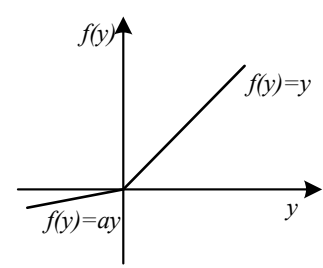

(b)PRelu
Fig. 4 ReLu vs PReLU

\section{EXPERIMENT}

\section{A. Public Palmprint Database}

Classic Palmprint recognition technology is mainly based on 2D Palmprint, which is convenient to collect and contains rich texture information. Therefore, we use 2D Database in PolyU 2D+3D Palmprint Database. Multispectral Palmprint can obtain more abundant Palmprint features and a higher recognition effect, because use the spectrum of different wavelengths, skin absorption and reflectance is different, you can collect multiple bands of palmprint images, and these images are not easy to be forged. So we used the PolyU Multi - Spectral Palmprint Image Database.

1) PolyU 2D+3D Palmprint Database: PolyU 2D $+3 \mathrm{D}$ Palmprint Database [14] contains 8000 sample images collected from 400 different palms. Samples of each palmprint were collected in two separate sessions, and 10 samples were collected in each experiment. The average time interval between the two sessions was one month. In the experiment, samples taken from the same palm can be regarded as the same class, and samples taken from different palms can be regarded as different classes. Therefore, this sample base contains a total of 400 classes, with each class containing 20 samples. Each sample contains its 3D ROI (area of interest) and corresponding 2D ROI. This article uses the part of 2D ROI in the database, and the image size is $128 \times 128$.

2) PolyU Multi-Spectral Palmprint Database: PolyU Multi-Spectral Palmprint Image Database [15] collected 250 people left hand and right hand, a total of 500 classes of sample. Each palm was collected in two separate sessions, each time 6 palmprint images were collected, and the average time interval between the two sessions was 10 days. Each collection was done under lighting conditions of four different spectra, Red, Green, Blue, and Near Infrared, to collect palmprint images of four bands. The specific four bands are near-infrared (band I, wavelength $880 \mathrm{~nm}$ ), red (band R, wavelength 660nm), green (band G, wavelength $525 \mathrm{~nm}$ ), and blue (band $\mathrm{B}$, wavelength $470 \mathrm{~nm}$ ). Therefore, there are a total of $4 \times 12 \times 500=24000$ palmprint images of all palms in each band. Using the above palmprint processing method, the ROI area of $128 \times 128$ palmprint was obtained for the next experiment.

\section{B. Experimental Environment}

The training equipment used in this article includes an eight-core Intel i7 processor, an NVIDIA1060 graphics card, and $16 \mathrm{~GB}$ of memory. The experiment of this paper is carried out in the open source machine learning library Tensorflow2.0 of Google, and the programming language is Python3.5.

\section{Experiments Results}

In order to test and select the appropriate learning rate, we selected 2D Database in the Palmprint Database of PolyU $2 \mathrm{D}+3 \mathrm{D}$ for the experiment. We selected all 400 classes of data in the database. After data preprocessing, we divided the data into $60 \%$ training set, $20 \%$ test set and $20 \%$ verification set to compare the performance.

We tested the influence of different learning rates on the recognition rate with 2500 iterations, and the results are shown in table II. After comprehensive experiment comparison and selection, we found that the convergence rate and training time synthesis reached the best speed and the maximum recognition rate when the recognition rate was 0.0008 .

We divided all the data in the database into $60 \%$ training set, $20 \%$ test set and $20 \%$ verification set. The determined learning rate and network structure are applied to conduct training on the PolyU $2 \mathrm{~d}+3 \mathrm{~d}$ Palmprint Database and PolyU Multi - Spectral Palmprint Database, the results are shown in table III below. In addition, classical methods such as LBP and HOG are used to extract features from the same training set, and classical algorithms such as KNN and SVM are used for training and classification recognition. Then, we use LDA to reduce the dimension of data after LBP and HOG feature extraction, and then conduct classification and recognition. All results are shown in table III below. 
TABLE II.

PERFORMANCE COMPARISON

\begin{tabular}{|c|c|c|c|c|c|}
\hline $\begin{array}{l}\text { Learnin } \\
\text {-g rate }\end{array}$ & Iterations & Loss & Time/s & $\begin{array}{c}\text { Accuracy } \\
\text { of test } \\
\text { data } \\
\end{array}$ & $\begin{array}{c}\text { Recog } \\
\text {-nition } \\
\text { rates } \\
\end{array}$ \\
\hline \multirow{3}{*}{0.01} & 500 & 1.5120 & 448.98 & $56.00 \%$ & \multirow{3}{*}{$\begin{array}{c}99.94 \\
\%\end{array}$} \\
\hline & 1000 & 0.0533 & 1352.85 & $90.00 \%$ & \\
\hline & 1500 & 0.0001 & 2461.74 & $100.00 \%$ & \\
\hline \multirow{3}{*}{0.001} & 500 & 0.7400 & 436.48 & $70.00 \%$ & \multirow{3}{*}{$\begin{array}{c}99.95 \\
\%\end{array}$} \\
\hline & 1000 & 0.0592 & 1428.42 & $92.00 \%$ & \\
\hline & 1500 & 0.0001 & 2561.06 & $100.00 \%$ & \\
\hline \multirow{3}{*}{0.0008} & 500 & 0.7454 & 378.42 & $90.00 \%$ & \multirow{3}{*}{$\begin{array}{c}99.96 \\
\%\end{array}$} \\
\hline & 1000 & 0.0147 & 1226.62 & $100.00 \%$ & \\
\hline & 1500 & 0.0026 & 2427.54 & $100.00 \%$ & \\
\hline \multirow{3}{*}{0.0005} & 500 & 0.9277 & 444.21 & $46.00 \%$ & \multirow{3}{*}{$\begin{array}{c}99.95 \\
\%\end{array}$} \\
\hline & 1000 & 0.0719 & 1242.87 & $96.00 \%$ & \\
\hline & 1500 & 0.0048 & 2449.93 & $100.00 \%$ & \\
\hline \multirow{3}{*}{0.0003} & 500 & 1.5656 & 441.12 & $40.00 \%$ & \multirow{3}{*}{$\begin{array}{c}99.56 \\
\%\end{array}$} \\
\hline & 1000 & 0.1695 & 1236.46 & $86.00 \%$ & \\
\hline & 1500 & 0.0025 & 2431.89 & $98.00 \%$ & \\
\hline \multirow{3}{*}{0.0001} & 500 & 2.4503 & 437.07 & $78.00 \%$ & \multirow{3}{*}{$\begin{array}{c}99.64 \\
\%\end{array}$} \\
\hline & 1000 & 0.6367 & 1227.33 & $74.00 \%$ & \\
\hline & 1500 & 0.3356 & 2288.35 & $92.00 \%$ & \\
\hline
\end{tabular}

TABLE III.

TEST RESOULT

\begin{tabular}{|c|c|c|c|c|c|c|}
\hline \multirow{2}{*}{\multicolumn{2}{|c|}{ Methods }} & \multicolumn{5}{|c|}{ Recognition rate of Spectras or Databases (\%) } \\
\hline & & Red & Blue & Green & NIR & Sub2D \\
\hline Alexnet & & 99.99 & 99.99 & 99.97 & 99.86 & 99.96 \\
\hline $\begin{array}{c}\text { LBP+ } \\
\text { KNN }\end{array}$ & \multirow{2}{*}{$\begin{array}{l}\mathrm{N} \\
\mathrm{O}\end{array}$} & 88.35 & 89.55 & 91.44 & 87.10 & 90.31 \\
\hline $\begin{array}{c}\text { LBP+ } \\
\text { SVM }\end{array}$ & & 90.25 & 77.40 & 83.20 & 93.10 & 92.88 \\
\hline $\begin{array}{c}\text { HOG+ } \\
\text { KNN }\end{array}$ & \multirow{2}{*}{$\begin{array}{l}\mathrm{L} \\
\mathrm{D} \\
\mathrm{A}\end{array}$} & 97.90 & 98.40 & 95.95 & 96.45 & 97.31 \\
\hline $\begin{array}{c}\text { HOG+ } \\
\text { SVM }\end{array}$ & & 99.45 & 99.75 & 98.60 & 99.30 & 98.58 \\
\hline $\begin{array}{c}\text { LBP+ } \\
\text { KNN }\end{array}$ & \multirow{4}{*}{$\begin{array}{l}\mathrm{L} \\
\mathrm{D} \\
\mathrm{A}\end{array}$} & 91.60 & 87.80 & 88.25 & 92.75 & 94.31 \\
\hline $\begin{array}{c}\text { LBP+ } \\
\text { SVM }\end{array}$ & & 89.50 & 87.40 & 89.10 & 93.45 & 93.81 \\
\hline $\begin{array}{l}\text { HOG+ } \\
\text { KNN }\end{array}$ & & 99.65 & 99.83 & 99.35 & 99.35 & 99.84 \\
\hline $\begin{array}{c}\text { HOG+ } \\
\text { SVM }\end{array}$ & & 99.60 & 99.45 & 98.30 & 99.10 & 99.25 \\
\hline
\end{tabular}

It can be seen from the experiment that the method of deep learning can get a better recognition rate in each spectrum and database, which also proves the effectiveness of the method of deep learning in palmprint recognition. At the same time, the experiment also proves that in the aspect of palmprint recognition, the feature extraction and learning ability of deep learning is slightly stronger than the classic algorithm combination of HOG, LBP, KNN and SVM.

\section{CONCLUSION}

The classical palmprint recognition method needs a series of tedious operations such as feature extraction, feature selection and classifier selection, and has great limitations in the process of feature extraction and feature selection. Compared with the classical algorithm, the convolutional neural network has the ability to directly input the image and then obtain the classification result, and it has a good nonlinear fitting ability. When using convolutional neural network for palmprint recognition, we do not need to construct feature extraction algorithm. In this paper, a classic convolutional neural network Alexnet is used for palmprint recognition, and its recognition rate can reach up to $99.99 \%$ on test data, which is another idea of palmprint recognition.

\section{ACKNOWLEDGMENT}

This work is supported by National Natural Science Foundation (No. 61673316), and Major Science and Technology Project of Guangdong Province (No. 2015B010104002).

\section{REFERENCES}

[1] Jain A. K., Ross A., Prabhakar S, "An introduction to biometric recognition," IEEE Transactions on Circuits \& Systems for Video Technology, vol.14, pp.4-20, Jan. 2004. doi:10.1109/TCSVT.2003.818349

[2] Unar J A, Seng W C, Abbasi A, “A review of biometric technology along with trends and prospects," Pattern Recognition, vol.47,pp.2673-2688, August. 2014.doi:10.1016/j.patcog.2014.01.016

[3] Kong A, Zhang D, Kamel M, "A survey of palmprint recognition," Pattern Recognition, 2009, vol.42, pp.1408-1418, July. 2009.doi: 10.1016/j.patcog.2009.01.018

[4] Wu Y P, Tian J W, Xu D, et al. "Palmprint Recognition Based on RB K-means and Hierarchical SVM," International Conference on Machine Learning \& Cybernetics, 2007. doi:10.1109/ICMLC.2007.4370778

[5] Kumar A, Bhargava M, Gupta R, et al. "Palmprint Authentication Using Pattern Classification Techniques," International Conference on Swarm, 2011.doi:10.1109/CIS.2007.106

[6] Li, Y.f, and Y. Zhang. "Palmprint recognition based on weighted fusion of DMWT and LBP," International Congress on Image \& Signal Processing, 2011. doi: 10.1109/CISP.2011.6100392

[7] Jia W, Gui J, Hu R X,"Palmprint Recognition Using Kernel Spectral Regression Discriminant Analysis and HOG Representation," International Workshop on Emerging Techniques \& Challenges for Hand-based Biometrics, 2010. doi: 10.1109/ETCHB.2010.5559288

[8] Hong D, Liu W, Jian S,"A novel hierarchical approach for multispectral palmprint recognition," Neurocomputing, vol.151, pp.511-521, March 2015 doi: 10.1016/j.neucom.2014.09.013

[9] Krizhevsky A, Sutskever I,Hinton G E, "Imagenet classification with deep convolutional neural networks," Advances in neural information processing systems, pp.1097-1105, January 2012. doi: $10.1145 / 3065386$

[10] Wang M, Deng W, “Deep face recognition: A survey,” arXiv preprint arXiv:1804.06655, 2018. doi:10.1109/SIBGRAPI.2018.00067

[11] Jalali A, Mallipeddi R, Lee M," Deformation Invariant and Contactless Palmprint Recognition Using Convolutional Neural Network," International Conference on Human-agent Interaction, 2015. doi:10.1145/2814940.2814977

[12] Zhang, D.; Kong, W.; You, J.; Wong, M,'Online palmprint identification," IEEE Trans. Patt. Anal. Mach. Intell, vol.25, pp. 1041-1049, Sept. 2003. doi:10.1109/TPAMI.2003.1227981

[13] He K, Zhang X, Ren S, "Delving Deep into Rectifiers: Surpassing Human-Level Performance on ImageNet Classification, "2015 IEEE International Conference on Computer Vision (ICCV). doi:10.1109/ICCV.2015.123

[14] W. Li, D. Zhang, L. Zhang, G. Lu, and J. Yan, " 3-D Palmprint Recognition with Joint Line and Orientation Features, "IEEE Transactions on Systems, Man, and Cybernetics, Part C, vol.41,pp.274-279, March 2011. doi: 10.1109/TSMCC.2010.2055849

[15] David Zhang, Zhenhua Guo, Guangming Lu, etc., "An Online System of Multi-spectral Palmprint Verification", IEEE Transactions on Instrumentation and Measurement, vol. 59, pp. 480-490, Feb. 2010. doi: 10.1109/tim.2009.2028772 